\title{
Evidence for Galaxy Formation at High Redshift
}

\author{
Tom Shanks, Nigel Metcalfe, Dick Fong \\ Physics Department, Durham University, Science Laboratories, South \\ Road, Durham DH1 3LE, UK \\ Henry McCracken \\ LAS, Traverse du Siphon, Les Trois Lucs, F-13102 Marseille, France
}

\author{
Ana Campos \\ Instituto de Matematicas y Fisica Fundamental, CSIC, Spain \\ David Thompson \\ California Institute of Technology, MS 320-47, Pasadena, CA 91125, \\ $U S A$
}

\begin{abstract}
Metcalfe et al. $(1995,1996)$ have shown that galaxy counts from the UV to the near-IR are well-fitted by simple evolutionary models where the space density of galaxies remains constant with look-back time while the star-formation rate rises exponentially. We now extend these results, first by using data from the Herschel Deep Field to show that these same models give detailed fits to the faint galaxy $r-i: b-r$ colour-colour diagram. We then use these models to predict the number counts of high redshift galaxies detected by the Lyman break technique. At $z \approx 3$ there is almost exact agreement between our prediction and the data, suggesting that the space density of galaxies at $z \approx 3$ may be close to its local value. At $z \approx 4$ the space density of bright galaxies remains unchanged; however, the space density of dwarf galaxies is significantly lower than it is locally, suggesting that we have detected an epoch of dwarf galaxy formation at $z \approx 4$. Finally, significant numbers of Lymanbreak galaxy candidates are also detected at $z \approx 6$ in the Hubble and Herschel Deep Fields; taking this observation together with a number of recent detections of spectroscopically confirmed $z \approx 6$ galaxies suggests that the space density of bright galaxies at $z \approx 6$ remains comparable to the local space density, and thus that the epoch of formation of bright galaxies may lie at yet higher redshift.
\end{abstract}

\section{Introduction}

The epoch of galaxy formation has been the subject of much recent controversy. Initially, observational evidence for a late epoch of galaxy formation appeared to come from analysis of the Canada-France Redshift Survey and the Hubble 
Deep Field, where the universal star-formation rate (SFR) appeared to peak at $z \approx 1$ (Lilly et al. 1995; Madau et al. 1996). However, it was also suggested that the CFRS/HDF data could still be consistent with models (Bruzual \& Charlot 1993) with a constant space density of galaxies whose SFR rises exponentially to redshifts $z \gtrsim 2$ (Metcalfe et al. 1996; Shanks et al. 1998). Indeed, if these 'pure luminosity evolution' models are modified by assuming a small amount of dust internal to spirals and a dwarf-dominated IMF for early-type galaxies, then they appeared to give very good fits to galaxy number count data from the UV to the near-infrared, at least in the case of low $\Omega_{0}$ (Metcalfe et al. 1996). The recent detection of high redshift galaxies as sub-mm sources (Smail et al. 1997; Hughes et al. 1998; Barger et al. 1998) also appears to support the idea that the SFR may rise to much higher redshifts. The 'pure luminosity evolution' models with internal spiral dust absorption have also been shown to be capable of producing fits to the sub-mm galaxy counts and the optical/near-IR galaxy counts simultaneously (Busswell \& Shanks 2000). Details of further constraints from the UV colours of galaxies on the dust extinction law and its evolution with redshift are given by Metcalfe et al. (2000).

In the Einstein-de Sitter Universe, with its smaller volume, it has been known for some time that such simple models only fit the optical counts to $B \lesssim 25$ mag (Yoshii \& Takahara 1988; Guiderdoni \& Rocca-Volmerange 1990; Koo et al. 1993) before underestimating the counts at fainter magnitudes. In this case, a new population of faint galaxies must be postulated; these extra galaxies could be the result of a process of dynamical merging at high redshift (Kauffmann \& Charlot 1998). Alternatively, as we assume here, they could be due to a population of 'disappearing dwarfs', denoted 'dE', that are luminous at high redshift but then dim dramatically to be virtually undetectable at low redshift (Babul \& Rees 1992; Metcalfe et al. 1996, 2000)

\section{Galaxy Counts and Colours}

We now test how well these models fit new ultra-deep optical and IR count data from HDF-N (Williams et al. 1996; Thompson et al. 1999), HDF-S (Gardner et al. 2000; Williams et al. 2000) and the William Herschel Deep Field (WHDF). Full details of our analyses of the Hubble and Herschel Deep Fields are given elsewhere (Metcalfe et al. 1995, 1996, 2000). The new optical $\left(R_{A B}\right)$ counts from HST STIS observations in the HDF-S are shown in the lower part of Fig. 1. The STIS passband is centred at $5850 \AA$, and has a width of $\sim 4000 \AA$. The fieldof-view is $50 \times 50 \operatorname{arcsec}^{2}$. The exposure time was $43 \mathrm{hrs}$; these are the deepest optical galaxy counts, reaching beyond $R_{A B}=30$. Also shown are the HDF-N,$\mathrm{S}$ WFPC-2 $R$ counts. Above these in Fig. 1 are the new $H$-band counts from NICMOS observations in HDF-N,-S. The field-of-view was $\sim 50 \times 50 \operatorname{arcsec}^{2}$. The exposure time was $36.5 \mathrm{hrs}$ for HDF-N and $48.3 \mathrm{hrs}$ for HDF-S; these are the deepest galaxy counts in the near-IR so far produced. Fig. 1 also shows the WHDF $H$ counts from Calar Alto 3.5-m observations plus $H$ counts from other authors. The models shown are those of Metcalfe et al. $(1996,2000)$ applied to both passbands. The no-evolution models generally underestimate the optical data. The $\mathrm{q}_{0}=0.05$ and $\Omega_{\Lambda}=0.7$ evolutionary models give excellent fits to the faintest limit of the HST STIS $R$ counts. The $\mathrm{q}_{0}=0.5+\mathrm{dE}$ dwarfs 


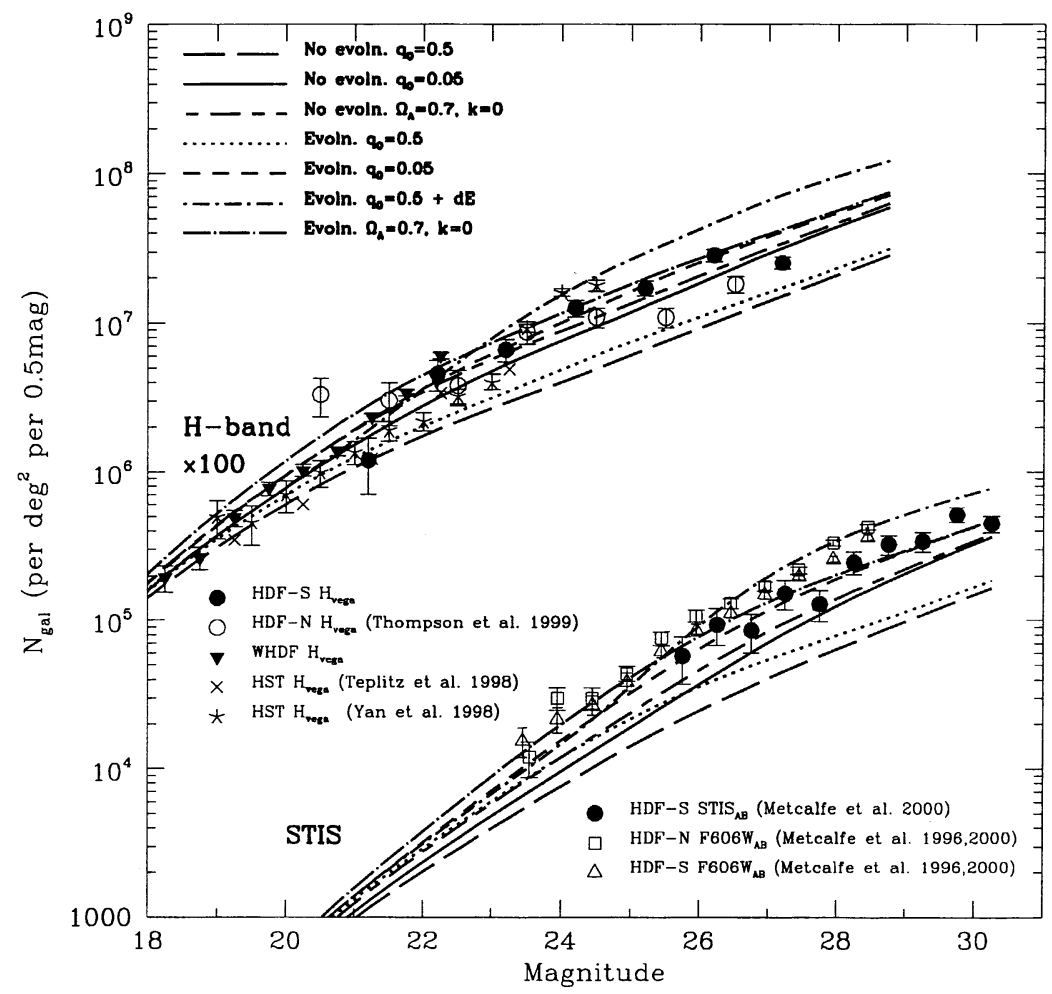

Figure 1. Lower points: HST STIS Hubble Deep Field South galaxy counts in the Clear passband from the data of Williams et al. (2000), analysed by techniques described elsewhere (Metcalfe et al. 2000). Also shown are the HST WFPC-2 $R$ (F606W) counts in the HDF-N,-S fields; the STIS counts are slightly lower than, but still statistically consistent with, these data in the range of overlap. Upper points: HST NICMOS HDF-N,-S galaxy count data in the infra-red $H$ (F160W) passband from our analysis (Metcalfe et al. 2000) of the data of Williams et al. (2000) in the case of HDF-S and directly from Thompson et al. (1999) in the case of HDF-N. The counts have been scaled upwards by a factor of 100 for clarity. HST NICMOS data from other authors are also shown (Teplitz et al. 1998; Yan et al. 1998). The WHDF data comes from a $20 \mathrm{hr}$ exposure at the Calar Alto $3.5-\mathrm{m}$ telescope using the $\Omega^{\prime}$ camera. The field-of-view is $7^{\prime} \times 7^{\prime}$ and the galaxy counts reach $H=22.5$. The models shown are those of Metcalfe et al. (1996) applied to both passbands (see text). 
model includes the 'disappearing dwarf' galaxy population previously invoked to improve the galaxy count fit of the $\mathrm{q}_{0}=0.5$ evolutionary model (Metcalfe et al. 1996) and this model also remains consistent with the new, fainter data. This model slightly overestimates the faintest counts at $R_{A B} \gtrsim 29$ and $H \gtrsim 25$, possibly indicating a slightly smaller contribution from the $\mathrm{dE}$ population than assumed here (Metcalfe et al. 1996). Nevertheless, we also regard this model as remaining in reasonable agreement with the ultra-deep galaxy count data. At $H$, where the non-evolving models lie closer to the data than in the optical, all the evolving models except the $\mathrm{q}_{0}=0.5$ models without the $\mathrm{dE}$ component, provide a reasonable fit to the counts to the faintest $H \sim 28$ limit of the HST NICMOS data. We conclude that the predictions for the evolutionary models continue to agree with the data to these new, very deep completeness limits of $R_{A B}=30$ and $H=28$.

Beyond $R_{A B} \approx 26$ and $H \approx 23$ we are looking down the luminosity function of galaxies and seeing intrinsically faint galaxies $\left(\mathrm{M}_{H} \sim-12\right)$, which on consideration of their colours are mostly at $z \approx 2$ (Metcalfe et al. 1996, 2000). Thus intrinsically faint galaxies at relatively low redshifts dominate the galaxy number counts at the faintest limits. In the low $q_{0}$ case, the excellent fit of the evolutionary models suggests that the slope of the faint galaxy luminosity function at $z \approx 2$ may be consistent with that measured locally. The need for the extra dwarf population to fit the optical counts in the $q_{0}=0.5$ case, suggests that here the luminosity function slope must steepen by $z \approx 2$ to match the faint count data.

Finally, we compare the model predictions for the $r-i: b-r$ colour-colour diagrams with the data from the WHDF. Fig. 2 shows how the predictions show a distinctive plume of early-type galaxies which first rise from $b-r \approx 1.5, r-i \approx 0.7$ to redder $b-r$ colours before turning bluewards again, as the increasing SFR at high redshift begins to affect the redshifted UV light. In the case of the spirals, a horseshoe-shaped curve is predicted reaching out to $z \approx 3$ at its faint limit. The agreement between models and data in both cases is quite remarkable, suggesting that for all their simplicity these models share the essential qualities required by the data; for example, the faint galaxies seem to divide into just two evolutionary classes corresponding to early and late types as we assume. And the parameter differentiating the evolution in each class appears to be the starformation rate, with an exponential e-folding time of $\tau=2.5 \mathrm{Gyr}$ for early-types and $\tau=9$ Gyr for spirals.

\section{3. 'Drop-out' Galaxies}

We now use these models to predict the number counts of galaxies detected by the Lyman break technique (Steidel et al. 1999) at $2 \lesssim z \lesssim 7$. We first consider the $z \approx 3$ range, where the data comes from detecting the Lyman break galaxies via the UV dropout technique in ground-based data (Steidel et al. 1999; Metcalfe et al. 2000) and at fainter limits from the Hubble Deep Fields N,S (Madau et al. 1996; Metcalfe et al. 2000). In Fig. 3a we then simply compare the observed ground-based number counts of the $z \approx 3$ galaxies with the model predictions, with the same comparison for the HST-based data being shown in Fig. $3 \mathrm{~b}$. We see that in both cases at $z \approx 3$ there is excellent agreement between the three 


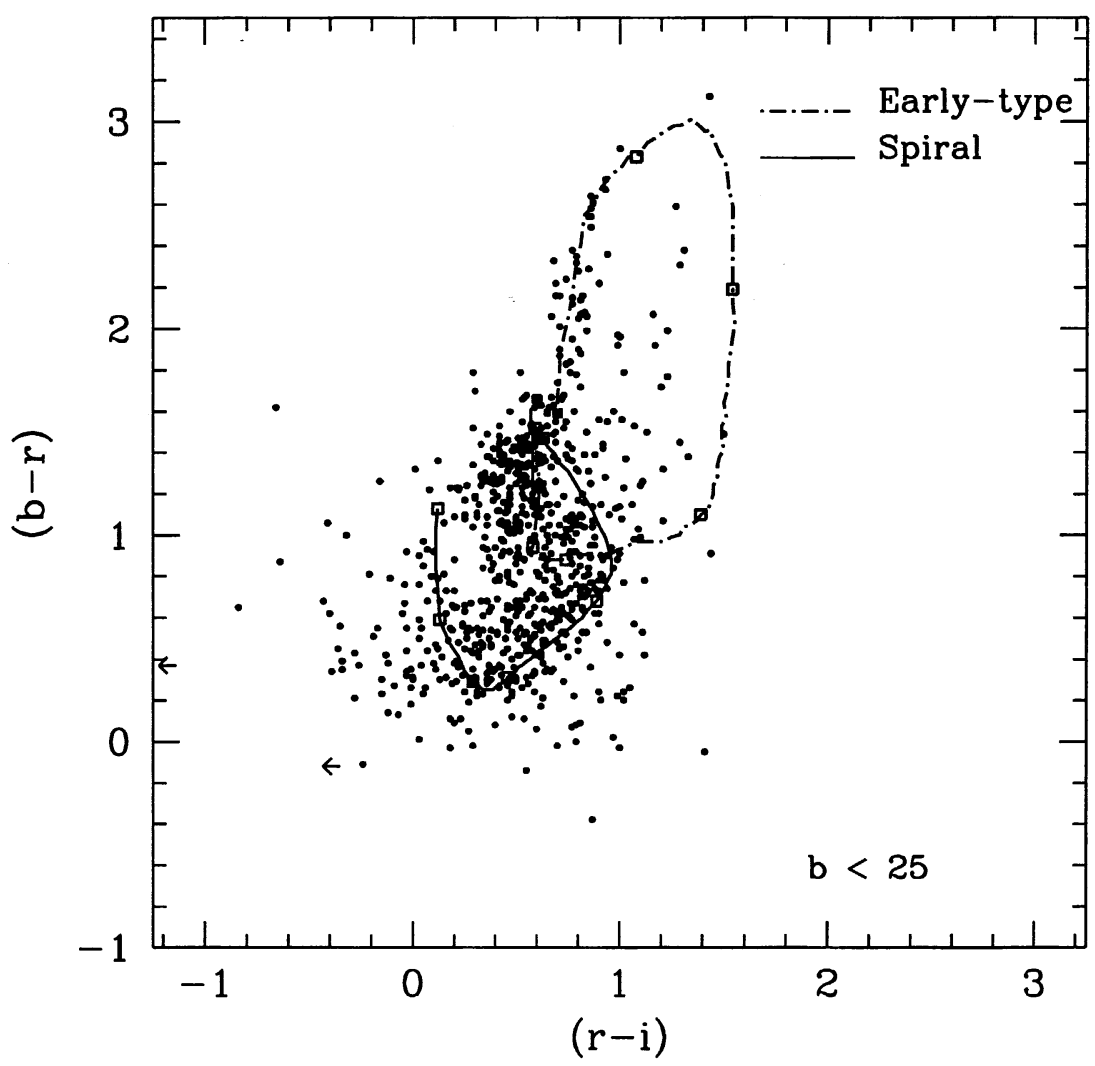

Figure 2. The $B$-limited $r-i: b-r$ colour-colour diagram from the Herschel Deep Field data, showing the evolutionary tracks predicted from our Bruzual \& Charlot models for essentially two galaxy types for $0 \lesssim z \lesssim 3$. Boxes indicate $0.5 z$ intervals. A plume of early-type galaxies can be seen which rise from $b-r \approx 1.5, r-i \approx 0.7$ to redder $b-r$ colours before turning bluewards again, as increasing star-formation at $z \gtrsim 0.5$ begins to affect the redshifted UV light. The spirals follow a horseshoe-shaped track below the early-types. This result clearly shows that there are just two main forms for the evolutionary behaviour of faint galaxies and the excellent fit of the Bruzual \& Charlot models suggests that the main distinguishing feature is the star-formation rate, with $\tau=2.5 \mathrm{Gyr}$ assumed here for the early-types and $\tau=9 \mathrm{Gyr}$ for the spirals. 

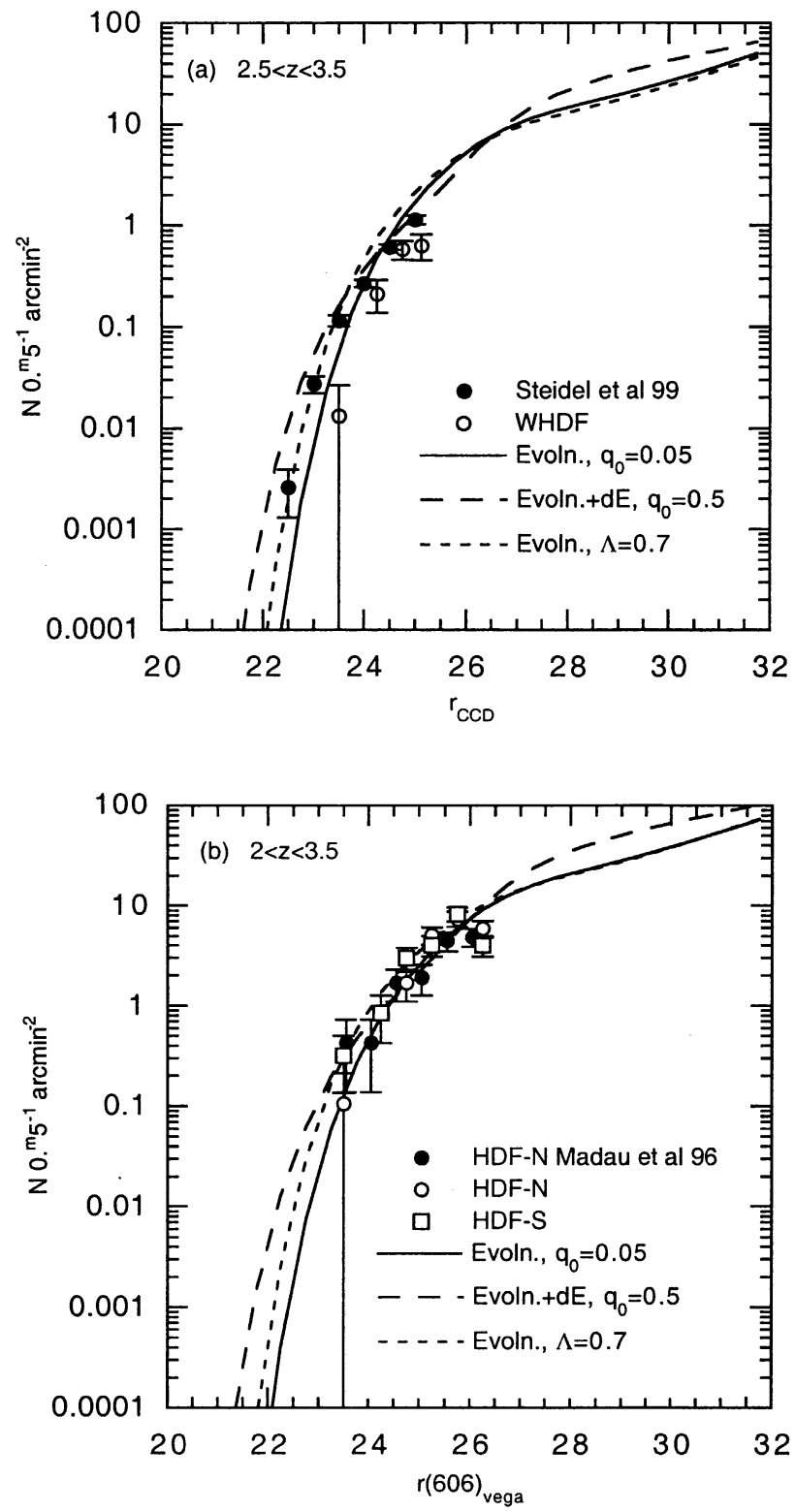

Figure 3. (a) The $r_{C C D}$ number count of $2.5 \lesssim z \lesssim 3.5$ ground-based UV dropout galaxies compared to our evolutionary model predictions. In the $\mathrm{q}_{0}=0.5+\mathrm{dE}$ case the contribution of the $\mathrm{dE}$ population can be seen at $r>25$. The WHDF data reasonably agrees with the data of Steidel et al. (1999) and overall we regard the agreement between the three evolutionary models and the data as excellent. (b) The $r$ $\left(\mathrm{F} 606 \mathrm{~W}_{\text {vega }}\right)$ number count of $2 \lesssim z \lesssim 3.5 \mathrm{HDF}$ UV-dropout galaxies compared to the same models as in (a). Again the agreement between these fainter data and the three evolutionary models is excellent. 

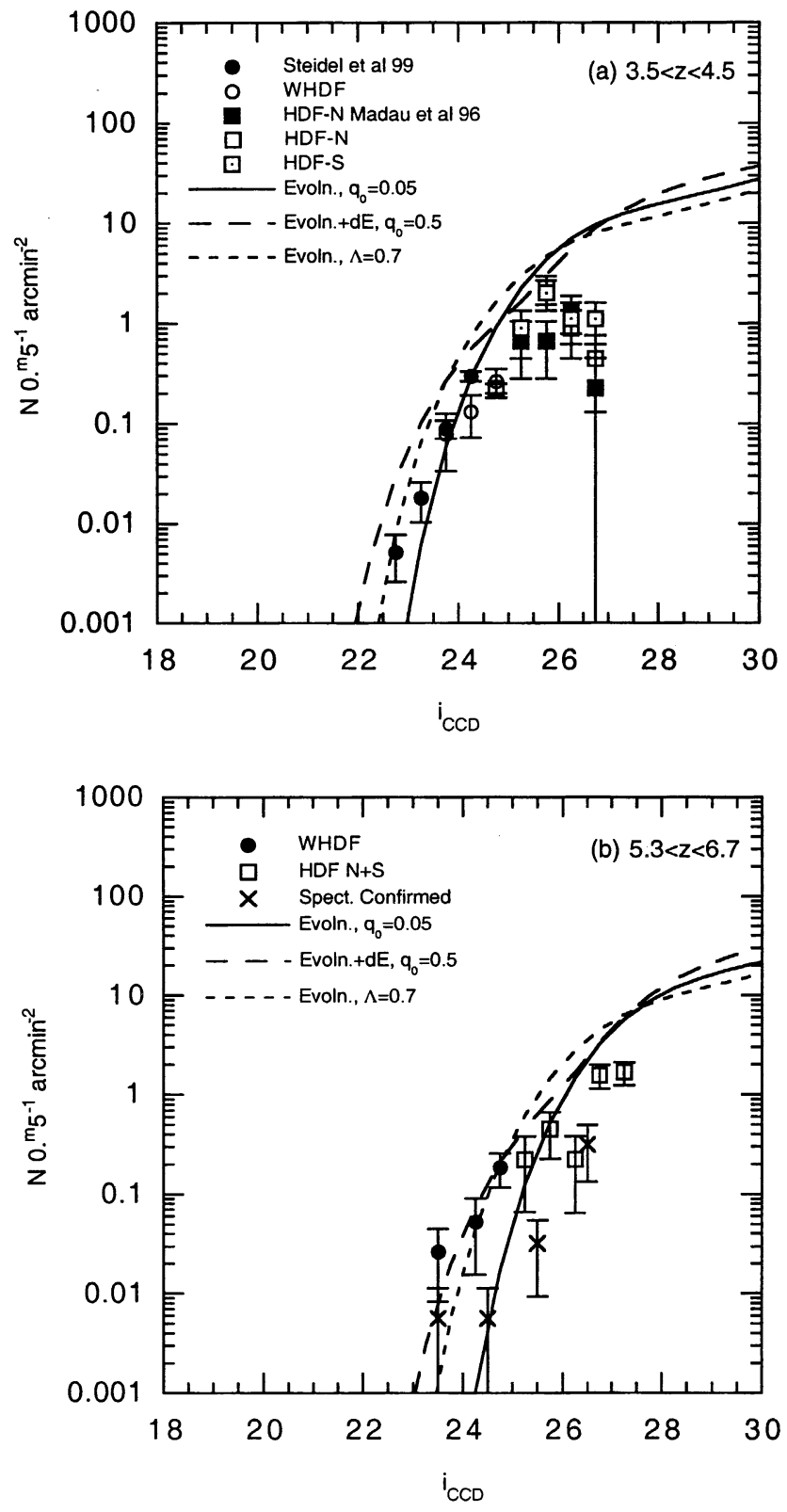

Figure 4. (a) The $i_{C C D}$ number count of $3.5 \lessgtr z \lesssim 4.5 B$-dropout galaxies from the WHDF and HDF data, compared to our model predictions. The models fit well at bright magnitudes but are $5 \times$ too high at faint magnitudes, indicating that luminous galaxies may form at $z \gtrsim 4$ while dwarfs form at $z \lesssim 4$. (b) The $i_{C C D}$ number count of $5.3 \lesssim z \lesssim 6.7 R$-dropout galaxies from the WHDF and HDF data (Metcalfe et al. 2000). The crosses are based on 6 spectroscopically confirmed $z \approx 6$ galaxies. The models show good agreement for the brighter galaxies, indicating that luminous galaxies form at $z \gtrsim 6$. 
basic evolutionary models and the data. Thus, models which were last adjusted to fit the Keck $B<24 \mathrm{n}(z)$ at $z \approx 1$ are now found to give a strikingly good fit to what is effectively the galaxy luminosity function at $z \approx 3$. Since it is the spirals which dominate the bright part of the model luminosity functions at $z=3$, this means that the Lyman break galaxies might be identified with the bright end of the evolved spiral luminosity function. This result is consistent with the flat SFR: $z$ relation between $1 \lesssim z \lesssim 3$ found by Steidel et al. (1999) and the slightly rising SFR: $z$ relation predicted by the models in this range (Metcalfe et al. 2000); the models predict only a small amount of evolutionary brightening in the range $1 \lesssim z \lesssim 3$ and the constant space density of spiral galaxies then ensures the SFR:z relation remains fairly flat. In the fainter, HST-based data in Fig. 3b, the models continue to fit well. However, it should be noted that the $\mathrm{q}_{0}=0.5$ model relies on the contribution of the 'disappearing dwarfs' to maintain agreement with the faint count data; it can be seen that, without this $\mathrm{dE}$ contribution, the $\mathrm{q}_{0}=0.5$ model would start to underestimate the counts.

In Fig. 4 a we now compare the number count of $z \approx 4$ Lyman break galaxies detected by $B$ dropout to the same models. At bright magnitudes, where the data comes mainly from Steidel et al. (1999), the models appear to fit the data well, suggesting that the bright spirals may have been in place even as early as $z \approx 4$. However, at fainter magnitudes where the data comes from the HDF-N,$\mathrm{S}$ and the WHDF, the simple pure luminosity evolution models at last appear to be breaking down, with the models generally overestimating the $z \approx 4$ faint galaxy luminosity function. The observed faint $\left(i_{C C D}>24.5\right)$ galaxy count falls below our predictions by a factor of $\sim 5$, indicating that while bright $\mathrm{L}^{*}$ galaxies may have been in place at $z \approx 4$, the faint galaxy population may have formed in the range $3 \lesssim z \lesssim 4$. Steidel et al. (1999) also noted that the galaxy luminosity function at $z \approx 4$ appeared flatter than at $z \approx 3$ but speculated that this may be due to the HDF-N being an unrepresentative field. The fact that the same result is seen in the WHDF and HDF-S argues that this result may be real. The result also explains why the SFR density of Steidel et al. (1999) appears to be constant between $3 \lesssim z \lesssim 4$ when measured from bright ground-based data, but appears to fall at $z \approx 4$ when measured from the fainter HDF data.

In Fig. 4b we extend this approach, now comparing the number counts of $z \approx 6$ Lyman break galaxies detected by $R$ dropout in the HDF and WHDF to the same models. We have taken galaxies with $r-i>1.5$ and $i-H<2.2$ in the WHDF and (F606-F814) vega $>2.44$ in the HDF-N,-S to define consistently the redshift range $5.3 \lessgtr z \lesssim 6.7$ or $z \approx 6$ as suggested by the models. Again we see that the observed number density of bright WHDF galaxies is well fitted by the models. The fainter galaxies from the HDF remain a factor of $\sim 5$ lower than predicted. Since the HDF data probes less deeply into the galaxy luminosity function than at $z \approx 4$, this is consistent with a further decrease in the number of faint galaxies relative to the local density. The crosses in Fig. $4 \mathrm{~b}$ show the number counts we have compiled from 6 galaxies with Keck or HST spectroscopy (Dey et al. 1998; Weymann et al. 1998; Spinrad et al. 1998; Chen et al. 1998). It can be seen that although there is reasonable agreement at the faint end with our unconfirmed HDF-N,-S R dropout candidates, at the bright end there are somewhat fewer galaxies detected. This disagreement may be partly due to the difficulty of detecting Ly- $\alpha$ emission against an increasingly bright sky at these longer wavelengths and partly due to contamination of our WHDF candidates 
by lower redshift early-type galaxies. However, the fact that a population of spectroscopically confirmed $z \approx 6$ galaxies have already been identified and that even larger numbers of $R$ dropout candidates exist, again suggests that significant numbers of bright galaxies had formed by $z \approx 6$, pushing the epoch of formation of giant galaxies back to even higher redshifts.

\section{Conclusions}

Thus, the picture of galaxy formation which is suggested is that bright galaxies form first at $z \gtrsim 4$ and then faint galaxies form later at $3 \lesssim z \lesssim 4$. This is similar to the 'downsizing' scenario suggested in other data at $z \approx 1$ (Cowie et al. 1997) and may possibly be connected to claims of evolving, faint luminosity function slopes at even lower redshifts (Brinchmann et al. 1998). This scenario is unexpected in hierarchical galaxy formation theories where dwarf galaxies are expected to form earlier than giant galaxies. In a CDM model with feedback, this order may be reversed with supernovae winds blowing gas out of dwarf galaxies preferentially because of their shallower gravitational potentials. However, suppression of star formation at $z \gtrsim 1$ is usually required in such models to produce the observed luminosity function at the present day (Brinchmann et al. 1998); it thus remains to be seen how well a CDM model will deal with the large numbers of luminous galaxies detected between $4 \lesssim z \lesssim 7$. New observations of the galaxy luminosity function at even higher redshifts are now required to determine the redshift of formation of the brightest galaxies.

\section{Acknowledgments}

We gratefully acknowledge G. Bruzual and S. Charlot for use of their isochrone synthesis code. We also acknowledge the use of the Hubble Space Telescope Deep Field data. We further acknowledge use of the Calar Alto 3.5-m telescope and the $\Omega^{\prime}$ Camera. The WHT is operated on the island of La Palma by the Isaac Newton Group at the Spanish Observatorio del Roque de los Muchachos of the Instituto de Astrofisica de Canarias. H. J. McCracken and N. Metcalfe acknowledge PPARC funding.

\section{References}

Babul, A., \& Rees, M. J. 1992, MNRAS, 255, 346

Barger, A. J., et al. 1998, Nature, 394, 248

Brinchmann, J., et al. 1998, ApJ, 499, 112

Bruzual, G., \& Charlot, S. 1993, ApJ, 405, 538

Busswell, G. S., \& Shanks, T. 2000, MNRAS, in press, astro-ph/0002081

Chen, H. W., Lanzetta, K. M., \& Pascarelle, S. 1998, Nature, 398, 586

Cowie, L. L., Hu, E. M., Songaila, A., \& Egami, E. 1997, ApJ, 481, L9

Dey, A., et al. 1998, ApJ, 498, L93

Gardner, J. P., et al. 2000, AJ, 119, 486 
Guiderdoni, B., \& Rocca-Volmerange, B. 1990, A\&A, 227, 362

Hughes, D. H., et al. 1998, Nature, 394, 241

Kauffmann, G., \& Charlot, S. 1998, MNRAS, 297, L23

Koo, D. C., Gronwall, C., \& Bruzual G. A. 1993, ApJ, 415, L21

Lilly, S. J., et al. 1995, ApJ, 455, 108

Madau, P., et al. 1996, MNRAS, 283, 1388

Metcalfe, N., Shanks, T., Fong, R., \& Roche, N. 1995, MNRAS, 273, 257

Metcalfe, N., et al. 1996, Nature, 383, 236

Metcalfe, N., et al. 2000, MNRAS, in press, astro-ph/0010153

Shanks, T., et al. 1998, in ASP Conference Series Vol. 146, The Young Universe:

Galaxy Formation and Evolution at Intermediate and High Redshift, ed.

S. D’Odorico, A. Fontana, \& E. Giallongo (San Francisco: ASP), 102

Smail, I., Ivison, R. J., \& Blain, A. W. 1997, ApJ, 450, L5

Spinrad, H., et al. 1998, AJ, 116, 2617

Steidel, C. C., et al. 1999, ApJ, 519, 1

Teplitz, H. I., Gardner, J. P., Malumuth, E. M., \& Heap, S. R. 1998, ApJ, 507, L17

Thompson, R. I., et al. 1999, AJ, 117, 17

Weymann, R. J., et al. 1998, ApJ, 505, L95

Williams, R. E., et al. 1996, ApJ, 112, 1335

Williams, R. E., et al. 2000, ApJ, submitted

Yan, L., McCarthy, P. J., Storrie-Lombardi, L. J., \& Weymann, R. J. 1998, ApJ, 503, L19

Yoshii, Y., \& Takahara, F. 1988, ApJ, 326, 1

\section{Discussion}

Eli Dwek: What contribution do ULIRGs make to your cosmic star formation rate?

Tom Shanks: None, because in our galaxy evolution models the dominant contributors to the cosmic star formation rate are evolving spirals with a small amount of dust. At high redshift these can be identified with the Lyman-break galaxies. Busswell and Shanks (2000) have shown that these evolving spirals can make 10 times more contribution to the sub-mmm background than unevolved spirals. However, these galaxies cannot explain the brightest sub-mm sources, and here Gunn and Shanks (2000) have suggested that ULIRGs in the form of obscured AGN may have a role to play.

Jim Peebles: I am impressed by the similarity of the star formation rate as a function of time in your model and from estimates based on UV luminosities. I assume you would predict a feature in the star-formation rate at $z>5$ from the formation of the early-type galaxies and spheroids of spirals.

Shanks: In our models, early-type galaxies (E/SO/Sab) are assumed to have a dwarf dominated IMF $(x=3)$. This was invoked to prevent the early types from overpredicting the numbers of $z>1$ galaxies seen in $K<19$ galaxy 
redshift surveys. Also, a Salpeter IMF $(x=1.35)$ requires $\tau=1.5$ Gyr to fit the $r-i: b-r$ diagram, and this model then also overpredicts the number of $z>1$ galaxies at $B<24$. This means that the UV luminosity caused by the initial burst for $\mathrm{E} / \mathrm{SO} / \mathrm{Sab}$ galaxies is much reduced with respect to spirals, and results in our global SFR always being spiral dominated.

Alberto Franceschini: How does your model account for the evidence that there is a substantial change in galaxy morphology at about $z=2$, in the sense of a disappearance of large galaxies at large redshift?

Shanks: We agree that even a passive, pure luminosity evolution model produces too many $z \approx 1$ early-type galaxies in $K<19.5$ redshift surveys. This could be evidence for dynamical merging at $z \approx 1$, but early-type galaxies at this redshift still appear old. We therefore have assumed a steeper IMF for early types which reduces the passive evolution and produces agreement with the $\mathrm{K}$ redshift survey data. As long as the slope flattens again around 0.5 solar mass, it is difficult to reject this IMF from the spectra of local early type galaxies. 